\title{
The Canonical Expression of the Drama Product Manufacturing Processes
}

\author{
Dieter Van Rijsselbergen \\ ELIS - Multimedia Lab \\ Ghent University - IBBT \\ Gaston Crommenlaan 8/201 \\ B-9050 Ghent, Belgium \\ Dieter.VanRijsselbergen \\ @UGent.be
}

\author{
Barbara Van De Keer \\ ELIS - Multimedia Lab \\ Ghent University - IBBT \\ Gaston Crommenlaan 8/201 \\ B-9050 Ghent, Belgium \\ Barbara.VanDeKeer \\ @UGent.be
}

\author{
Rik Van de Walle \\ ELIS - Multimedia Lab \\ Ghent University - IBBT \\ Gaston Crommenlaan 8/201 \\ B-9050 Ghent, Belgium \\ Rik.VandeWalle@UGent.be
}

\begin{abstract}
As the broadcast industry is evolving toward IT-based facilities, the production workflows and their associated production metadata should similarly take advantage of IT commodities. This paper presents a manufacturing system for the production of drama television and motion picture programmes, constructed using IT-based technologies in a filebased media environment. This drama production facility implements a production workflow based on common industrial manufacturing processes and extensively models the individual aspects of the drama production process. We aim to show that the different processes contained in this manufacturing workflow can be expressed in terms of elementary building blocks, the canonical processes of media production. By identifying recurring and canonical functionality, process implementations can be simplified and input and output from different processes can be coordinated for better integration with external systems.
\end{abstract}

\section{Categories and Subject Descriptors}

H.1 [Models and Principles]: General; H.4 [Information Systems Applications]: General

\section{General Terms}

Design, Human Factors, Standardization

\section{Keywords}

IT-based broadcasting, drama production, product manufacturing, workflows, processes

\section{INTRODUCTION}

Throughout the broadcast industry, information technology (IT) is gradually replacing conventional broadcast technologies. Most notably, media production facilities are reengineering existing tape-based workflows to file-oriented approaches for acquisition, storage and processing of audio- visual material $[11,7]$. However, the transformation of only the audiovisual essence chain is not sufficient for the implementation of efficient IT-based workflows. The workflow information generated and gathered during the production, the so-called metadata, should become an integral of the production process [15] and should be modeled and employed as such by production systems.

Conventional production of drama, in particular, comprises sequential steps of rather ad hoc production that is usually still supported by a considerable and informal paper information flow. A lot of this information is used inefficiently and in an unstructured way, so that no automated system can take advantage of its semantics, leaving manual processing as an only option. Additionally, most tools available are concerned only with a limited subset of the production process and fail at appropriately integrating artifacts from external systems. By introducing a reliable and structured data model that models and centralizes all production workflow information and metadata, and by employing a formal definition of the processes involved in media production, optimal use can be made of an IT-centric drama production platform. A similar feat was demonstrated by Nack et al. in [14] for news production environments.

In the context of the IBBT project FIPA (File Integrated Production Architecture $)^{1}$ we have investigated how IT-based broadcast systems can significantly enhance existing production workflows for the creation of audiovisual drama. This paper presents the resulting proof-of-concept drama production system, the production processes it implements, and their expression as a function of the canonical processes of media production, introduced by Hardmann et al. [6]. The system architecture is introduced in Section 2 and the production processes are laid out in Section 3. Section 4 presents the canonical functionality identified in each of the production processes. Finally, Section 5 concludes on the work presented in this paper and briefly states a number of future research tracks.

\section{OVERVIEW OF THE FIPA DRAMA PRODUCTION SYSTEM}

Illustrated in Figure 1 is the architecture of the FIPA drama production system, targeted at the creation of daily soap operas, quality prime time drama series and even motion pictures. The architecture combines tools necessary to cover

\footnotetext{
${ }^{1}$ https://projects.ibbt.be/fipa/
} 


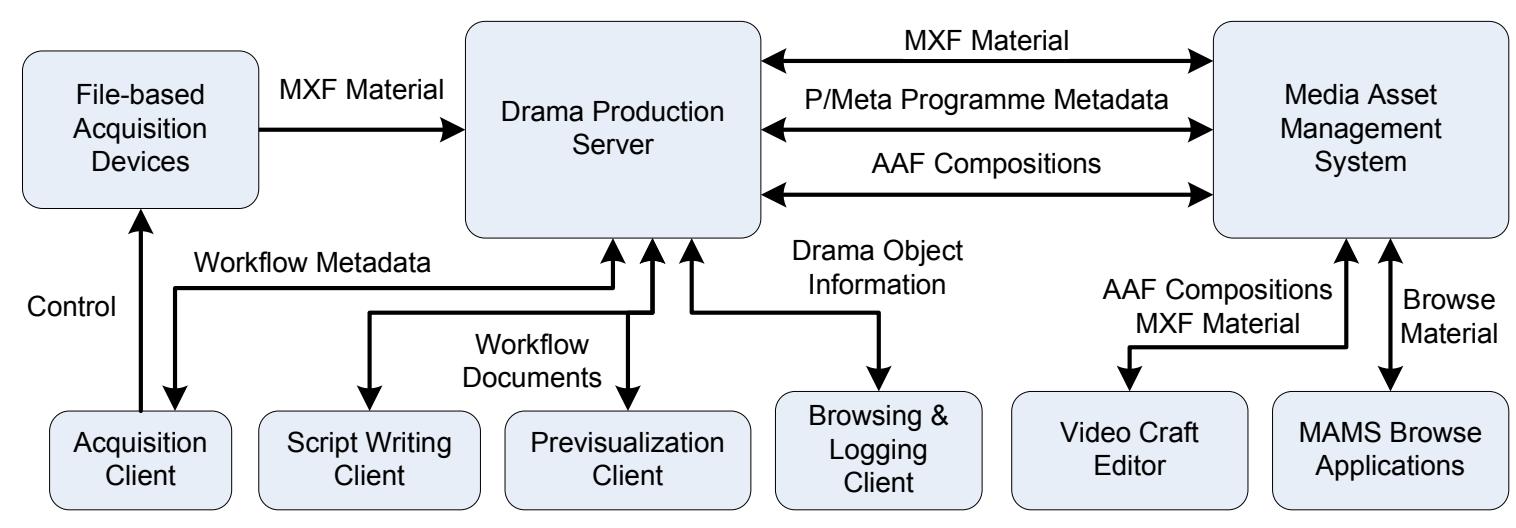

Figure 1: The FIPA drama production system architecture.

the entire drama production workflow, which conventionally consists roughly of pre-production (preparation of synopsis and dialogue scripts), production (acquisition and logging), and finally, post-production (editing and effects) [2]. The system is constructed in such a way that it provides abstraction from underlying components and integrations. This allows the entire system to function as a unified platform, enabling the implementation of media production processes that span entire productions from concept to final assembly.

The core of the system consists of a commercially available Media Asset Management System (MAMS) [12] and the FIPA drama production server. The MAMS functions as the central media hub for the entire production facility and its range of production tools. Material browsing, rough cut editing and play-out are tasks that are delegated through the MAMS. The MAMS deals with the integration of proprietary craft editing tools for video and audio editing. Through this integration, the MAMS exposes generic media editing interfaces to external systems, offering an abstraction of the underlying systems of which the specifics vary widely between vendors. This setup represents a common practice in IT-based broadcasting, as described in literature $[11,12]$ and deployed by some broadcasters, including project partner V.R.T. [7].

In order to provide functionality beyond the generic media production capabilities of the MAMS, the FIPA drama production server was implemented. This server provides additional integration with file-based capture devices and provides a backend for applications that use appropriate modeling of the extensive drama workflow. Such applications include custom production tools (a.o., a script editor, a pre-visualization tool and a video acquisition tool integrated with file-based capture devices), as well as database and file transferring services. A more detailed description of the FIPA drama system architecture is provided in [3].

\section{THE DRAMA PRODUCT MANUFACTURING PROCESS}

The architecture described allows for the construction of a system that spans and controls the entire media production process. In order to realize this, we have devised a metadata model and a production process model. The data model represents objects from the entire drama production process. It also structures and centralizes all workflow information so

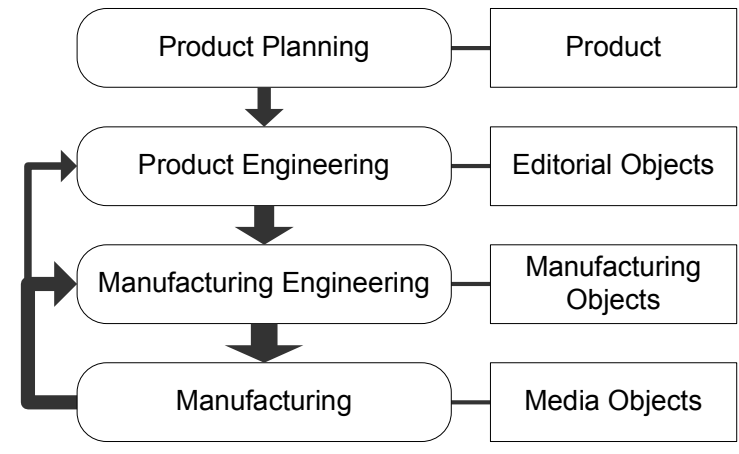

Figure 2: The drama manufacturing production process. Processes are shown left, the associated data model objects on the right.

that full advantage can be taken of the semantics within this information by every component in the system [17].

While investigating current methods of drama production (in literature $[2,8]$, and by visiting actual productions), we found that many preparatory steps often precede the recording of any amount of audiovisual material. A lot of attention is spent on the research and development of the story, resulting in elaborate descriptions of scene actions and settings. Directors often prepare a meticulous planning of shooting setups and provide estimated montage decisions, possibly assisted by sketches or storyboards for visualization. These preparations are motivated by a matter of cost: the production of drama is a complex and expensive endeavor, and accurate planning can help to reduce unexpected expenses. Inspired by these findings, the FIPA drama production system employs a media production workflow that is modeled after common industrial production workflows, where a product is assembled from components that go through a number of different stages of production during its inception, starting with initial product planning and product engineering, manufacturing engineering, and finally, the actual process of manufacturing or assembly [10]. This workflow is illustrated in Figure 2. These abstract process classes provide a formalized and integrated framework for actually implemented production processes to collaborate in. Note that this manufacturing process model is meant to serve as an underlying 
structure and should not be visible to actual (human) users of the system. All production processes implemented for users still represent methods commonly used by drama staff today and are supported by tools that provide familiar interfaces using drama terminology and concepts.

The following three subsections define the different manufacturing process classes, and include examples in the form of concrete processes implemented by the FIPA system. Each section also lists the primary data model objects the processes discussed operate on. Subsection 3.4 introduces the process feedback mechanism and Subsection 3.5 briefly mentions the evaluation of our system by production people.

\subsection{Product Planning \& Engineering}

A typical product in drama media production is a fictional series consisting of episodes, but it can also be a single episode, or even a motion picture. The lifecycle of such a product is formally started in the Product Planning process. This process determines an initial set of requirements for the product, including format, genre, subject, number of episodes and perhaps even coarse story lines. The product planning process is typically orchestrated from an external Enterprise Resource Planning (ERP) system which feeds this product information into the drama system. We classify whatever notion the ERP system has about the product as the Product object, but this is of no further concern to the drama system.

Within the drama system, the Product Planning specifications are passed onto the Product Engineering process. This process determines product specifications while maintaining abstraction from the actual media asset manufacturing process. For drama, this process defines the narrative aspect of the product. Currently, two specific drama Product Engineering processes are defined: synopsis writing and dialogue script writing. In both processes, producers, story writers and editors, researchers and the director collaborate on the definition of the narrative aspects of the product. A synopsis contains only a summary of a scene or episode, while the dialogue script contains integral character performance descriptions and dialogue. Within the system, these specifications are stored as XML Schema-compliant documents, such that their structure and semantics can be made understandable to human and computing actors.

Product Engineering defines a product as a composition of logical units of creative or editorial work (e.g., episodes, sequences and scenes), each of which is represented by an Editorial Object in the data model. Of all defined Editorial Objects, scenes are the most prominent ones since they are the typical discrete unit of work in which drama is performed, shot and edited. The Product Engineering specifications that result from Product Engineering are bundled into Product Engineering documents and are associated with the Editorial Object, hence determining its editorial and narrative content.

\subsection{Manufacturing Engineering}

The Product Engineering specifications are used by Manufacturing Engineering to define the exact manufacturing guidelines as to how actual media assets will be realized. In fact, this process creates blueprints for the individual components that the final product will be assembled of, and specifies how this assembly is to be made. One very common type of Manufacturing Engineering built into our system is the definition of a shooting script. Before shooting, a director prepares this so-called shooting script, in which he specifies which camera and microphone points of view (POVs, informally referred to as 'shots') will be used to capture a scene. A more elaborate Manufacturing Engineering process is previsualization. By means of a rough three-dimensional model of the set and characters involved in a scene, the performance of the scene can be prepared using elementary character animation. This generates a visual idea of how a scene should be performed and shot. Previsualization uses the camera POVs defined by the director in the shooting script. As such, different camera angles can be sampled and tweaked, and even an idea of a rough cut montage can be constructed. The previsualization process adds information about scene locations, character animation and additional POV parameters to existing product specifications. This is similar to methods demonstrated in [5].

The final Manufacturing Engineering process implemented in the FIPA system is editing. During the editing process, existing media assets are cut and ordered into a presentation that tells exactly the story that the director and editor wish to express to the audience. The editing process results in a composition: a structured document which describes how the montage is to be assembled into an audiovisual media asset. Our system implements a number of editing processes, each suited for specific needs and users. Live Mixing is used on the set for real-time editing of multicamera television series and is performed on-the-fly as the scene is acted out. Because of elaborate stage and acquisition planning, this form of editing usually delivers montages that require only minor modifications afterwards. Secondly, Browse Editing allows directors and editors to create rough cuts by means of tools provided by the MAMS. This editing process uses low-resolution proxy versions of the original material and requires no expensive craft editing setup; a low-end client suffices. Finally, for larger and more extensive editing tasks, which possibly require specific image transitions or special effects, the drama production system also supports true Craft Editing, through the integration of the MAMS with proper craft editor tools. Both textual (XML) and binary (Advanced Authoring Format, AAF [1]) formats are used as document structures for compositions.

The Product Engineering documents are extended into Manufacturing Engineering documents, which contain detailed descriptions of how media assets are to be manufactured. These Manufacturing Engineering documents are in turn associated with the Editorial Object. Each document describes a number of components or Manufacturing Objects, the individual units of manufacturing work. Manufacturing Objects include Camera and Microphone POVs and Compositions.

\subsection{Manufacturing}

When the Manufacturing Engineering specifications have been finalized, the actual creation or Manufacturing process of the product, or components thereof, can be initiated. The result of this process are tangible audiovisual media assets. The acquisition of audiovisual material on the set, through filming or video capturing, is the most common Manufacturing process. The FIPA system implements acquisition by means of file-based capturing devices which expose recorded material directly via the network and wrapped in Material 
Exchange Format (MXF) [16] files. This allows these media asset containers to be automatically linked with the manufacturing engineering components and the scene they were manufactured for.

The rendering of a composition into an 'flattened' media asset is also a Manufacturing process, albeit one that is mostly used transparently when using a craft editor. A final Manufacturing process, Previsualization Rendering, translates $3-\mathrm{D}$ scene and animation descriptors into sequences of twodimensional images. The two latter processes illustrate that, given sufficiently accurate manufacturing engineering specifications, some manufacturing processes can be automated. While not currently implemented, another automated task would be real-time editing based on speech recognition and a shooting script with editing decisions.

The drama system keeps track of each created audiovisual media asset through a Media Object. Every Media Object is automatically related to one or more Manufacturing Objects: the Media Object is said to be a realization of the Manufacturing Object. This relation is enriched with creation metadata about the media asset: coding characteristics, image resolution, location and time of acquisition, etc.

\subsection{Feedback}

In a drama production environment the demands for flexibility and creativity are high. As such, engineering specifications must be updatable at any time in the production process to reflect creative and practical decisions taken by the drama crew. The need for engineering specification changes is typically triggered from Manufacturing processes. Figure 2 shows that Manufacturing processes can influence the engineering processes through the provided feedback channel. As such, feedback can trigger new media assets to be created differently (e.g., recording of a new take with different lighting parameters). In more severe cases, feedback can even prompt changes to Product Engineering specifications, e.g., a scene might be rewritten to better reflect the acting performances and mood of an already captured scene.

The feedback mechanism is also used to connect manufacturing processes of different components. For example, the feedback concerning the creation of a media asset is fed to the subsequent editing Manufacturing Engineering process that uses this asset in a montage.

The formal process definitions enable the system to enforce data model integrity, so that engineering changes do not violate existing data model or production planning dependencies (e.g., actor planning, budget allocations). In some cases, explicit approval or rejection of changes by a producer or director may be required before engineering changes are allowed to impact the system.

\subsection{Evaluation}

The FIPA drama production system has been demonstrated to production people on a number of occasions. The reduction of manual labor in the production process (e.g., no more handling of tapes, timecode registrations, paper annotations), integration of different production tools, as well as the availability and management of all interconnected production information from a centralized web application were met with enthusiasm. The concepts of our manufacturing engineering tools were understood and agreed upon, but were said to require improvement in order to be usable by most people on actual productions.

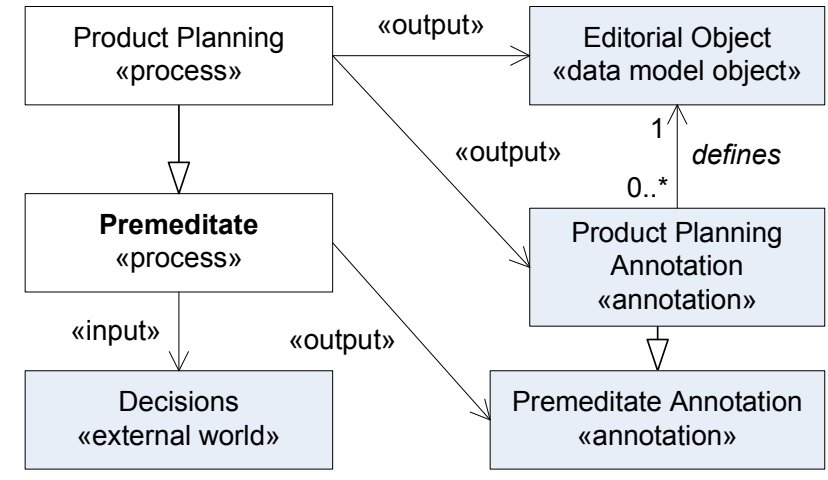

Figure 3: Canonical mapping of the Product Planning process.

\section{MAPPING PRODUCTION PROCESSES}

This section expresses the presented product manufacturing processes in terms of the canonical processes of media production. This mapping allows us to identify recurring and canonical functionality in each of the production processes, so that process implementations can be simplified and inputs and outputs from different production processes and different systems can be coordinated. The mapping will allow foreign systems, that have also been expressed in terms of canonical processes, to be more easily integrated with our drama production system. The remainder of this section presents the individual process mappings. Unified Modeling Language (UML) diagrams were employed to illustrate the relationships between the canonical processes and our manufacturing processes. The diagrams also show the input and output for each process. Table 1 lists the processes used in the FIPA system and their mapping to the canonical processes. The table also lists common process actors, which we omitted from the UML diagrams for conciseness.

\subsection{Product Planning}

The Product Planning process delivers product requirements (Product Planning Annotations) and an initial number of Editorial Objects, which at this early stage serve as placeholders for finished products (e.g., episodes, or the motion picture). Product Planning clearly represents the desire, of entities external to the system, for the creation of media assets. Hence, it subclasses the Premeditate canonical process. This is visualized in the UML diagram Figure 3.

\subsection{Product Engineering}

The Product Engineering process subdivides placeholder editorial objects into a number of individually manufacturable editorial objects (e.g., scenes) and adds new engineering specifications to existing and new editorial objects. Input is provided by the initial specification from Product Planning, and feedback from the Manufacturing Engineering and Manufacturing processes. The output of this process consists of these additional editorial objects and new or updated engineering specifications.

The functionality of three canonical processes is employed to implement Product Engineering: Premeditate, Construct Message and Annotate. Nack [13] and Kerherve et al. [9] have already identified the act of film script writing as a form of premeditation. In fact, this can be generalized to 
all of Product Engineering, which again serves as an act of premeditation. Product Engineering however also aims to convey a message by means of the product's narrative aspects, which it defines. Hence, Product Engineering specializes the Premeditate and Construct Message processes, as illustrated by Figure 4. In turn, Dialogue Script Writing and Synopsis Writing subclass Product Engineering.

Product Engineering specifications are added to an existing Editorial object and its specifications. Because the Premeditate and Construct Message processes only accepts external world influences, additional functionality is required. The Annotate process associates annotations with process artifacts. As such, new or updated specification annotations (i.e., Product Engineering Documents) may be appended to Editorial Objects. The Annotate process is another parent class of Product Engineering. In order to implement the feedback mechanism described in the previous section, process feedback annotations are accepted by the Product Engineering process. As such, decisions made during the Product Engineering process can be influenced. If required, this feedback can also be appended to the editorial object by Annotate functionality.

\subsection{Manufacturing Engineering}

The Manufacturing Engineering process is very similar to Product Engineering with respect to the canonical processes: the generic Manufacturing Engineering process specializes the Premeditate, Construct Message and Annotate processes. Construct Message is used again here, as ways in which media assets are created can also be used to convey messages to an audience. Again, output Manufacturing Engineering documents are associated with existing editorial objects through Annotation. Process feedback input is also present here. In fact, since more iterations of the Manufacturing Engineering-Manufacturing-cycle are likely to occur, it is even more important in this context. Each of the processes described in Subsection 3.2 is a specialization of the generic Manufacturing Engineering process. The editing process, however, also incorporates functionality of the canonical Organise process [13]. Naturally, the implemented editing processes Browse Editing, Live Mixing and Craft Editing are subclasses of the generic Editing process. The output of each Manufacturing Engineering process consists of Manufacturing Engineering Document annotations, containing engineering specifications. In the case of editing, these also include a Composition Document structure. The class diagram for this process is similar to the diagram depicted in Figure 4 so it was omitted for conciseness.

\subsection{Manufacturing}

The canonical Create Media Asset process, which uses the input of the Premeditate process in order to realize media assets, is a close fit to the Manufacturing process. Additionally, each of the Manufacturing processes discussed in Subsection 3.3 can subclass existing Create Media Asset specialization classes defined in [6]; the Acquisition process is a form of Capturing, the Previsualization Rendering is an act of Generation. Finally, Composition Rendering transforms one or more media assets into a new media asset; in simple cases only through a concatenation of media asset particles (i.e., frames or samples), but possibly also by applying various transitions or other effects. Hence, Composition Rendering is suited to subclass the Transforming process. In many cases, Composition Rendering results in a finished media asset, ready for consumption (by an editor and director during production, or even by an actual audience). As this fits the definition of the Publish process, namely adapting a structural document by selecting appropriate parts and possibly makes refinements before it is presented to a user in a final form, we add Publish as a parent class for Composition Rendering. Along with media assets, the Manufacturing process delivers creation metadata. This includes feedback (based on human or possibly automated evaluation) and is fed to the engineering processes. The other metadata comprises media asset and realization information and is linked to the created media object by Annotate. The class diagram associated with Manufacturing is shown in Figure 5.

\subsection{Supporting Processes}

Not all functionality of the drama production system is covered by the manufacturing processes alone. Other processes, often performing an interconnecting function between manufacturing processes or production systems, exist. In these supporting processes, canonical functionality can also be identified. This section briefly discusses these processes in terms of their parent canonical process. While this is not an exhaustive list of all supporting processes in the FIPA drama production system, the examples given are representative for processes not listed.

\subsubsection{Annotate}

The Annotate process is already used by the engineering and manufacturing processes. An additional logging process employs Annotate to allow additional remarks to be added to media assets later in the production process. For example, a director can add additional comments upon viewing the dailies for a specific scene. The annotations added by the Annotate process join the creation annotation as feedback for the Manufacturing Engineering and Product Engineering processes.

\subsubsection{Query}

A Query mechanism is implemented by each individual system used in the drama production system:

- Searching within the drama production application (e.g., searching through editorial objects, manufacturing parameters and realization information) by means of a browse client (i.e., a web browser accessing the webbased front-end of the drama production application).

- Searching through a MAMS interface, in various applications (e.g., to support material selection in the rough cut editing stage or to find promotional photographic material).

- Searching in the craft editor environment to retrieve dailies and stock footage for the editing process.

\subsubsection{Package}

Explicit and implicit packaging is used as a supporting process throughout the drama production cycle. This nonexhaustive list of packaging operations includes:

- Simultaneously captured media assets, each realizing a unique POV component, are packaged as a Media Object Group [17], which indicates a time relationship and allows the elements to be played as an isochronous and multi-dimensional unit. 


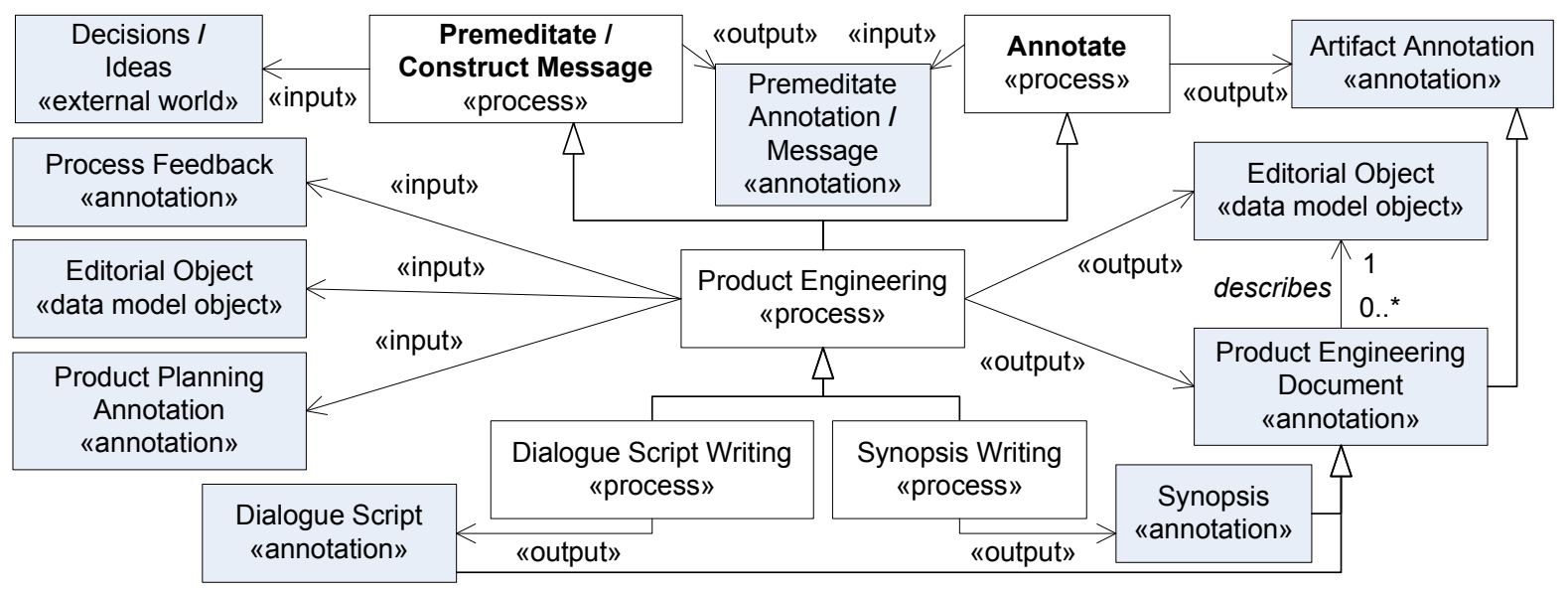

Figure 4: Canonical mapping of the Product Engineering process.

- During production, workflow metadata is exchanged between components of the system. Exchanges between the drama production server and the MAMS occur by means of the P/Meta [4] and AAF metadata standards (cfr. Figure 1). Packaging is used to wrap editorial object information and media object metadata into $\mathrm{P} /$ Meta documents, and to wrap compositions into AAF files.

- The entire dialogue script of an episode, which is to be distributed to actors on the set, can be considered a composite media asset. In this case, packaging can take place to wrap individual scene script fragments into a single episode dialogue script.

- Audiovisual media assets are wrapped into MXF container files to be transported between different systems in the facility. This occurs, for instance, when MXF files are transferred from file-based acquisition devices to the drama production system.

\subsubsection{Distribute}

As with Packaging, the functionality of the Distribute process is used in many places in the drama production process. Distribute is used for transfers of audiovisual material from one system to another within the production system. These can be transfers of authoritative full-resolution versions, but also of browse versions retrieved from the MAMS cache. Ubiquitous networking and file-based workflows allow for all major distribution tasks to be carried out over an interconnecting IT network. Further distribution processes concerned with the distribution of drama content to end user audiences is mostly an exclusive matter of the MAMS and play-out hardware or other distribution channels (e.g., DVD, video-on-demand). As such, their applicability falls outside the scope of our system.

\section{CONCLUSIONS AND FUTURE WORK}

In this paper we have introduced the FIPA system and its manufacturing processes for drama production, which allow for efficient use of IT-based media production and broadcasting environments. We have expressed the production processes in terms of the canonical processes of media production. We found the canonical processes of media produc- tion to be well suited to represent the functionality of our manufacturing-oriented drama production processes, which reify a more formal production workflow than is conventionally used. This successful mapping allows implementers of new manufacturing methods and of foreign integrating systems to better coordinate processes in terms of inputs, outputs and functionality. The production processes of the FIPA system currently represent only methods commonly used in drama production today. Future extensions of the system will focus on more elaborate modeling techniques for Product- and Manufacturing Engineering. This way, we hope to automate more of the production workflow and to simplify operations for production people involved. Additionally, we aim to extend the system with mastering processes for the adaptation of drama products to specific user platform capabilities and preferences, taking full advantage of the rich semantics already present in the engineering specifications constructed as part of the drama production workflow.

\section{ACKNOWLEDGMENTS}

The authors wish to thank Maarten Verwaest (V.R.T.) for the invaluable input concerning a manufacturing approach to media production. The research activities that have been described in this paper were funded by Ghent University, V.R.T., the Interdisciplinary Institute for Broadband Technology ( $50 \%$ co-funded by industrial partners), the Institute for the Promotion of Innovation by Science and Technology in Flanders (IWT), the Fund for Scientific ResearchFlanders (FWO-Flanders), the Belgian Federal Science Policy Office (BFSPO), and the European Union.

\section{REFERENCES}

[1] AMW Association. Advanced Authoring Format Object Specification v1.1. Apr. 2005. Available at http://www.aafassociation.org.

[2] S. Ascher and E. Pincus. The Filmmaker's Handbook. Penguin Putnam, Inc., New York, 1999.

[3] M. De Geyter, N. Oorts, and L. Overmeire. File-based broadcast workflows on MAM systems and their integration demands. In Proceedings of the SMPTE Technical Conference 200\%, October 2007. 


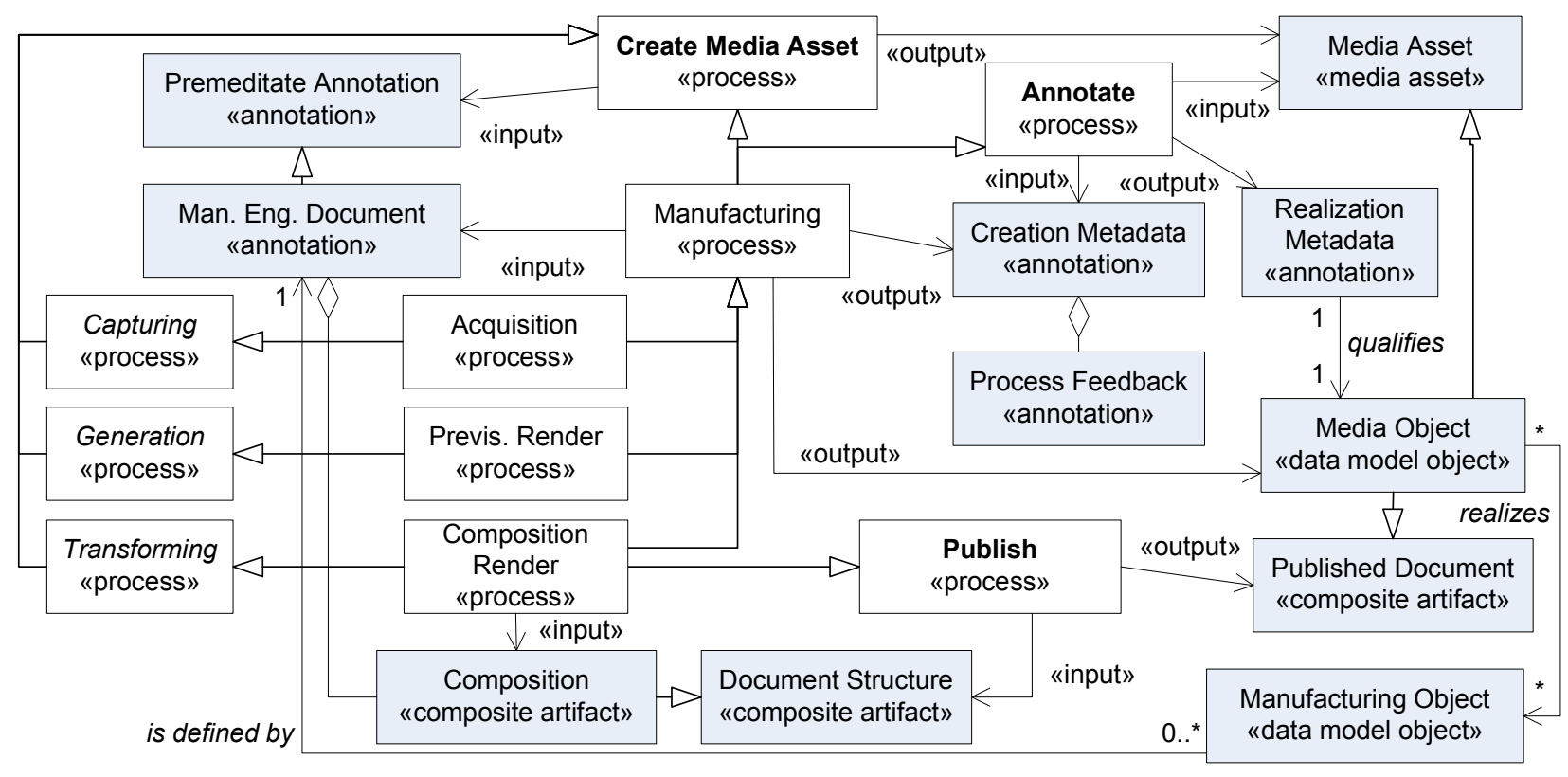

Figure 5: Canonical mapping of the Manufacturing process.

[4] EBU. P/Meta Metadata Exchange Scheme v1.1. Technical Report 3295. June 2005. Available at http://www.ebu.ch/en/technical/metadata/specifications/.

[5] O. Grau. A 3d production pipeline for special effects in tv and film. In Proceedings of Mirage 2005 Conference, Computer Vision/Computer Graphics Collaboration Techniques and Applications., March 2005.

[6] L. Hardmann, F. Nack, Z. Obrenovic, B. Kerherve, and K. Piersol. Canonical process of media production. In Proceedings of the ACM Workshop on Multimedia for Human Communications - From Capture to Convey (MHC 05), November 2005.

[7] J. Hoffman, P. Soetens, and M. De Geyter. An Integrated File-based Media Production Workflow: The Digital Media Factory. In Proceedings of the IBC 2007 Conference, September 2007.

[8] S. Katz. Film Directing: Shot by Shot: Visualizing from Concept to Screen. Michael Wiese Productions, Studio City, 1991.

[9] B. Kerherve, A. Ouali, and P. Landon. Design and production of new media artworks. In Proceedings of the ACM Workshop on Multimedia for Human Communications - From Capture to Convey ( $\mathrm{MHC}$ 05), November 2005.

[10] D. Koshal, editor. Manufacturing Engineer's Reference Book. Butteworth-Heinemann Ltd, Oxford, 1993.
[11] A. Kovalick. Video Systems in an IT Environment. Elsevier Inc, Oxford, 2006.

[12] A. Mauthe and P. Thomas. Professional Content Management Systems. John Wiley \& Sons Ltd, New York, 2004.

[13] F. Nack. Capture and transfer of metadata during video production. In Proceedings of the ACM Workshop on Multimedia for Human Communications - From Capture to Convey (MHC 05), November 2005.

[14] F. Nack and W. Putz. Saying what it means: Semi-automated (news) media annotation. Multimedia Tools and Applications, 22(3):263-302, March 2004.

[15] D. J. Rayers. Metadata in TV production: Associating the TV production process with relevant technologies. SMPTE Motion Imaging Journal, 112(9):287-292, September 2003.

[16] Society of Motion Picture Television Engineers. SMPTE Standard for Television - Material Exchange Format (MXF) - File Format Specification. SMPTE 377M-2004, 2004.

[17] D. Van Rijsselbergen, M. Verwaest, B. Van De Keer, and R. Van de Walle. Introducing the Data Model for a Centralized Drama Production System. In Proceedings of the IEEE Intl. Conference on Multimedia \& Expo 200\%, pages 615-618, July 2007. 
Table 1: Mapping of Drama Manufacturing processes and Canonical processes.

\begin{tabular}{|c|c|c|}
\hline Canonical Process & $\begin{array}{l}\text { Manufacturing } \\
\text { Process }\end{array}$ & Process \\
\hline Premeditate & Product Planning & $\begin{array}{l}\text { Specification of initial product requirements (1) } \\
\text { Input: Product requirements from outside the system (ERP) } \\
\text { Output: Editorial Objects, initial product specification annotations }\end{array}$ \\
\hline \multirow[t]{2}{*}{$\begin{array}{l}\text { Premeditate, } \\
\text { Construct Message, } \\
\text { Annotate }\end{array}$} & $\begin{array}{l}\text { Product } \\
\text { Engineering }\end{array}$ & $\begin{array}{l}\text { Synopsis Writing (2): by story writer and editor, researcher, producer } \\
\text { Input: Editorial Objects [1], initial product specification annotations [1], feedback [7, } \\
8,9,10] \\
\text { Output: Editorial Objects, Synopsis (Prod. Eng. Document) } \\
\text { Dialogue Script Writing (3): by story writer and editor, researcher, producer } \\
\text { Input: Editorial Objects }[1,2] \text {, initial product specification annotations [1], Synopsis } \\
\text { (Prod. Eng. Document)[2], feedback [7, } 8,9,10] \\
\text { Output: Editorial Objects, Dialogue Script (Prod. Eng. Document) }\end{array}$ \\
\hline & \multirow[t]{2}{*}{$\begin{array}{l}\text { Manufacturing } \\
\text { Engineering }\end{array}$} & $\begin{array}{l}\text { Shooting Scripting (4): by director, producer } \\
\text { Input: Editorial Objects [3], Dialogue Script (Prod. Eng. Document) [3], feedback [7, } \\
8,9,10] \\
\text { Output: Shooting Script (Man. Eng. Document), Manufacturing Objects } \\
\text { Previsualization (5): by director, scene and character animator, producer } \\
\text { Input: Editorial Objects [3], Dialogue Script (Prod. Eng. Document) [3], feedback [7, } \\
8,9,10] \\
\text { Output: Output: Animation and 3-D Set Descriptors (Man. Eng. Documents) } \\
\text { Remark: Calls upon functionality provided by Shooting Scripting and Editing for } \\
\text { Manufacturing Engineering tasks, and for Previz. Render and EDL Render for viewing } \\
\text { of previsualized material. }\end{array}$ \\
\hline $\begin{array}{l}\text { Premediate, } \\
\text { Construct Message, } \\
\text { Annotate, } \\
\text { Organise }\end{array}$ & & $\begin{array}{l}\text { Editing (all, incl., Browse Editing, Live Mixing, Craft Editing) (6): by editor, director } \\
\text { Input: Editorial Objects [3], Dialogue Script (Prod. Eng. Document) [3], feedback [7, } \\
8,9,10] \text {, list of media assets [11,12, 13] . } \\
\text { Output: Composition specifications, Manufacturing Objects (typically one, represent- } \\
\text { ing the composition itself). }\end{array}$ \\
\hline Create Media Asset & \multirow[t]{2}{*}{ Manufacturing } & $\begin{array}{l}\text { Acquisition (7): by director, producer, director of photography, drama actors, camera } \\
\text { operators, other staff } \\
\text { Input: Shooting Script [4] } \\
\text { Output: Media Assets, Realization Information Annotations, feedback } \\
\text { Previsualization Rendering (8): by previz. application or stand-alone rendering node } \\
\text { Input: Animation and 3-D set descriptors [5] } \\
\text { Output: Media Assets, Realization Information Annotations, feedback }\end{array}$ \\
\hline $\begin{array}{l}\text { Create Media Asset, } \\
\text { Publish }\end{array}$ & & $\begin{array}{l}\text { Composition Rendering (9): by craft editor application or stand-alone rendering node } \\
\text { Input: Composition specification (EDL) [6] } \\
\text { Output: Media Assets, Realization Information Annotations, feedback }\end{array}$ \\
\hline Annotate & N.A. & $\begin{array}{l}\text { Logging (10): Additional annotations in the drama production application: by direc- } \\
\text { tor, other crew } \\
\text { Input: Media Assets }[7,8,9] \text {, Realization Information Annotations }[7,8,9] \text {, additional } \\
\text { logging annotations }[10] \\
\text { Output: Additional logging annotations }\end{array}$ \\
\hline \multirow[t]{3}{*}{ Query } & \multirow[t]{3}{*}{ N.A. } & $\begin{array}{l}\text { Searches in Drama System (11): by possibly all of drama crew } \\
\text { Input: Inventory list of drama objects, user criteria } \\
\text { Output: List of applicable drama objects }\end{array}$ \\
\hline & & $\begin{array}{l}\text { Searches in MAMS (12): by possibly all of drama crew } \\
\text { Input: Inventory list of MAMS objects, user criteria } \\
\text { Output: List of applicable MAMS objects }\end{array}$ \\
\hline & & $\begin{array}{l}\text { Searches in Craft Editors (13): by editor, director } \\
\text { Input: Inventory list of objects available in craft editor, user criteria } \\
\text { Output: List of applicable objects }\end{array}$ \\
\hline \multirow[t]{4}{*}{ Package } & \multirow[t]{4}{*}{ N.A. } & $\begin{array}{l}\text { Creation of MOGs (14): cfr., Paragraph } 4.5 .3 \text {, by drama production application } \\
\text { Input: a list of Media Assets }[7,8,9] \\
\text { Output: a Media Object Group (composite artifact) }\end{array}$ \\
\hline & & $\begin{array}{l}\text { Wrapping of exchangeable production metadata (15): cfr., Paragraph } 4.5 .3 \\
\text { Input: Editorial Objects, engineering specifications, Media Objects, realization infor- } \\
\text { mation related to Media Objects, logging annotations }[1,2,3,4,5,6,7,8,9,10] \\
\text { Output: P/Meta and AAF documents }\end{array}$ \\
\hline & & $\begin{array}{l}\text { Script Packaging (16): cfr., Paragraph } 4.5 .3 \text {, by drama production application } \\
\text { Input: A set of Dialogue Scripts [3] from individual scenes } \\
\text { Output: A composed media asset containing individual Dialogue Scripts }\end{array}$ \\
\hline & & $\begin{array}{l}\text { MXF wrapping of audiovisual material (17), by a.o., acquisition devices, craft editor } \\
\text { Input: a Media Asset }[7,8,9] \\
\text { Output: an MXF-wrapped media asset file }\end{array}$ \\
\hline Distribute & N.A. & $\begin{array}{l}\text { Viewing (18): Throughout the production process, media assets are retrieved from } \\
\text { the drama system over the network for viewing by the production crew. } \\
\text { Input: a Media Asset }[7,8,9] \\
\text { Output: Display of the Media Asset in workcenter, browse client, ... } \\
\text { MXF Media Transfer (19): Transfer of high resolution media assets from one system } \\
\text { to another in the production system, by various system components } \\
\text { Input: an MXF-wrapped Media asset [17] } \\
\text { Output: Transfer and storage of the media asset from one system onto a (for this } \\
\text { system) external location. }\end{array}$ \\
\hline
\end{tabular}

